\title{
A Pressure Control Method for Emulsion Pump Station Based on Elman Neural Network
}

\author{
Chao Tan, ${ }^{1}$ Nan Qi, ${ }^{1}$ Xin Zhou, ${ }^{1}$ Xinhua Liu, ${ }^{1,2}$ Xingang Yao, ${ }^{1}$ Zhongbin Wang, ${ }^{1}$ and Lei Si ${ }^{1}$ \\ ${ }^{1}$ School of Mechatronic Engineering, China University of Mining \& Technology, Xuzhou 221116, China \\ ${ }^{2}$ Xuyi Mine Equipment and Materials R\&D Center, China University of Mining \& Technology, Huaian 211700, China \\ Correspondence should be addressed to Xingang Yao; jorn13183175595@126.com and Zhongbin Wang; wangzbpaper@126.com
}

Received 21 October 2014; Revised 17 February 2015; Accepted 23 February 2015

Academic Editor: Francesco Camastra

Copyright (c) 2015 Chao Tan et al. This is an open access article distributed under the Creative Commons Attribution License, which permits unrestricted use, distribution, and reproduction in any medium, provided the original work is properly cited.

In order to realize pressure control of emulsion pump station which is key equipment of coal mine in the safety production, the control requirements were analyzed and a pressure control method based on Elman neural network was proposed. The key techniques such as system framework, pressure prediction model, pressure control model, and the flowchart of proposed approach were presented. Finally, a simulation example was carried out and comparison results indicated that the proposed approach was feasible and efficient and outperformed others.

\section{Introduction}

As key equipment to ensure coal mine safety production and achieve high efficiency, emulsion pump station provides high pressure emulsion for hydraulic support and hydraulic jack. The control system performances of quick response and steady tracking have direct influence on moving speed and bracing force [1]. Therefore, reasonable control method of emulsion pump station is essential to the whole coal mine.

Though tremendous progress has been made, great fluctuations of hydraulic system pressure still exist in actual use [2], which brings safety dangers to the whole coal mine. The existing system pressure control of emulsion pump station is a constant-pressure control method based on PID pressure compensation, the principle of which is to set expected pressure values for different working systems. When measured pressure value is too low or too high, which means the value is beyond error control, they change control pump unit motor frequency so that it increases or reduces pressure output to make the system pressure return into the appropriate operating range [3-5]. Essentially this method is a type of pressure compensation based on pressure itself, which cannot meet the demand of control in real time. On one hand, the emulsion pressure should come to the point that hydraulic support can achieve right motions in coal mining [6]. On the other hand, unnecessary emulsion pressure can bring resource waste and great fluctuations in the system [7]. That is, reasonable pressure emulsion is the key issue. At the same time, as a typical local recurrent network, Elman neural network is widely used.

Bearing the above observations in mind, Elman neural network is used for emulsion pump station to control pressure. The effect of hydraulic supports motions on system pressure is analyzed based on the field data. Combining the motions time and pressure set, we can obtain reasonable expected pressure. The model can not only follow the load, but also save energy.

The paper is structured as follows: some related works are outlined based on literature in Section 2. The factors to impact emulsion pump station pressure change are analyzed in Section 3. The framework and key technologies are proposed, and the flowchart of proposed approach is designed in Section 4. A simulation example and some comparisons are put forward to validate the proposed approach in Section 5. Our conclusions are summarized in Section 6.

\section{Literature Review}

In this section, we try to list and summarize some recent papers which are relevant to the control methods of pressure 
system. Generally, scholars make efforts to control pressure from two aspects; one is to improve the performance of system to follow the load [8-10] and the other is to propose new algorithms [11-13] to realize pressure control.

2.1. Pump Station Control Methods. Nowadays, pressure control methods of hydraulic pump station have maturely developed. Because of difficult data obtaining and other reasons, pressure control of emulsion pump station needs to be improved. To save the energy, a logical method and electric design were provided to realize automatic control $[14,15]$. In [16], a fusion approach of fuzzy control and PID was applied in emulsion pump station which got perfect dynamic performance and no state error. Giving hardware structure and software design, Xu et al. [17] researched frequency-conversion speed-regulation system of emulsion pump station. In the respect of system improving, Yang [18] analyzed the state of unloading valve and recovery pressure to ensure the stability of emulsion pump station. With the same working principle, emulsion pump station transfers power with emulsion, while other pump stations use hydraulic oil. A remote pressure control system was designed for capillary pressure in [19], and the result brought possibility to the creation of superior automatic microinjection controllers. Umrao and Chaturvedi [20] studied a novel fuzzy control approach for load frequency control, which avoided large number of rules. Yang et al. [21] also got good robustness from applying adaptive fuzzy control technology to pressure control of a pressurizer and the results showed that the controller was effective for the kind of systems with nonlinear, complex, and imprecise mathematical model. We can see that introduction of intelligent algorithm makes pump station pressure control have broader prospects.

2.2. Neural Network Control. As artificial neural network has the function of simulating human thinking and incomparable superiority in terms of establishing nonlinear and experiential knowledge simulation models, it is widely used in many fields, such as time series problems [22], prediction of field emission [23], prediction of mechanic parts performance [24], hydraulic winch control [25], and PMLSM position tracking control [26]. Nowadays, recurrent neural network is one of the study hotspots in the field of control. First proposed by Elman in 1990 [27], Elman neural network was the typical local recursion delay feedback neural network [28].

2.3. Discussion. Although many approaches for pressure control systems have been presented in the above papers, they have a common disadvantage summarized as follows. With the above approaches, the pressure fluctuations are too large to realize continuous right motions of hydraulic support. Moreover, large pressure fluctuations would impact the whole hydraulic system, especially the hydraulic valve and pipe, which accelerate abrasion of key components and reduce life of the equipment.

In order to reduce the pressure fluctuations, a pressure control method for emulsion pump station based on Elman
TABLE 1: Coding table of hydraulic states.

\begin{tabular}{lc}
\hline States & Code \\
\hline No motions & 0 \\
Retracting top coal-wall support & 1 \\
Lowering the column & 2 \\
Hauling support & 3 \\
Raising the column & 4 \\
Extending top coal-wall support & 5 \\
Propelling and pushing & 6 \\
\hline
\end{tabular}

neural network is proposed and a simulation example is provided to verify the effect of proposed method.

\section{Emulsion Pump Station and Hydraulic Support}

Emulsion pump station pressure is influenced by both hydraulic support motions and its pressure loss. The pressure loss contains pipeline pressure loss and valve pressure loss. At different oil pressures, different pressure losses occur. But we see the pressure loss as a part of hydraulic support motions influence in this paper. That is, pump station pressure is totally influenced by support states. And support states influence varies with the variation of the oil pressure.

In order to make it convenient for the following, we encode hydraulic states in Table 1.

\section{The Proposed Pressure Control Approach}

This section tries to present a new method aiming at providing stable emulsion pressure. The section has three main parts and can be elaborated through the following subsections.

4.1. The Framework of Proposed Approach. The construction process of control system is shown as follows.

(1) We get groups of data from industrial field. Because of large quantity and noise impact, data preprocessing is done before applying to experiments which means dropping data beyond range.

(2) After Elman neural network programming, 5040 groups of data are chosen as sampling to train the network. The relationship between oil pressure difference and motion order is simulated, and dynamic motion difference value matrix is obtained.

(3) Giving operating conditions, we know motion order of hydraulic supports. When oil pressure is not equal to set pressure, the difference is returned to the network. According to the network, dynamic motion influence valve is obtained. Regulating the frequency of frequency converter to reduce difference valve, we can get steady oil pressure. Figure 1 shows the framework of proposed approach. 


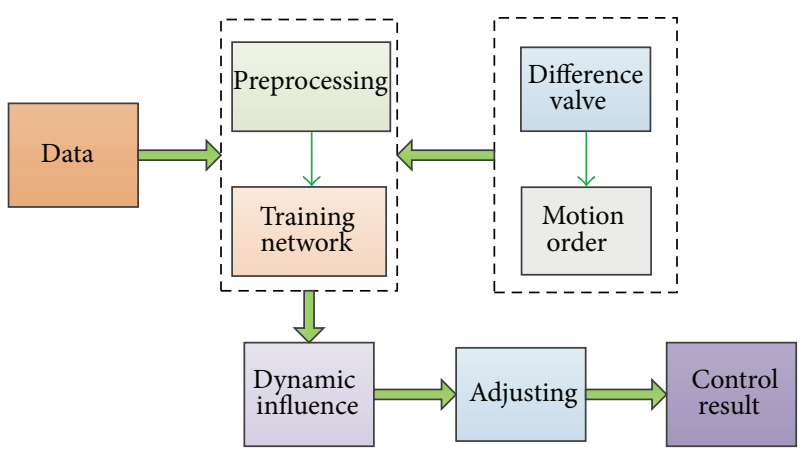

FIGURE 1: The framework of proposed approach.

4.2. Prediction of Emulsion Pressure. Neural network is an ideal type of nonlinear approximator. The important components are input layer, hidden layer, connected layer, and output layer. Compared with BP neural network, connected layer is added for partial feedback in Elman neural network [29]. Transfer function of it is linear, and to remember the state of past time series delay units are included. Having both memory unit and input of network as input of hidden layer, we can see dynamic memory function [30] in Elman neural network. Transfer function of connected and output layer is linear, while that of hidden layer is nonlinear, such as hyperbolic $S$ nonlinear function, step function, and prelinear function. Because of hidden layer receiving the data from input and memory data in connected layer, the same input can bring different output. The principle of Elman neural network with the characteristic of multi-input and single-output is shown in Figure 2.

Due to linear network, finite and discontinuous functions can be expressed in Elman neural network. For multi-input and single-output networks, we set $X_{c}$ as input of connected layer, $X_{h}$ as input of hidden layer, $Y_{h}$ as output of hidden layer, and $Y$ as exterior output of motion series $U$; then we get the formula of hidden layer input:

$$
X_{h}(k)=f\left(W^{1} X_{c 1}(k)+W^{2} U(k-1)+W^{4} X_{c 2}(k)\right),
$$

where $X_{c 1}(k)$ is output of connected layer 1 ,

$$
X_{c 1}(k)=\alpha_{y} X_{c 1}(k-1)+Y_{h}(k-1),
$$

and $X_{c 2}(k)$ is output of connected layer 2. Consider

$$
\begin{gathered}
X_{c 2}(k)=\gamma X_{c 2}(k-1)+Y_{h}(k-1), \\
Y(k)=g\left(W_{3} Y_{h}(k)\right) .
\end{gathered}
$$

In the formulas, $W^{1}$ is connected weight matrix of connected unit 1 and hidden layer, $W^{2}$ of input layer and hidden layer, $W^{3}$ of output layer and hidden layer, and $W^{4}$ of connecting unit 2 and hidden layer. $\alpha_{y}$ is the feedback parameter of connected unit 1, while $\gamma$ is that of connected unit 2. $f(\cdot)$ is the function of activation. Here we set $f(\cdot)$ as Sigmoid function [31]:

$$
f(x)=\frac{1}{1+e^{-x}} .
$$

If $y_{g}(k)$ is the output after the $k$ steps, error function can be expressed in Elman neural network:

$$
E(x)=\frac{1}{2}\left(y_{g}(k)-y(k)\right)^{T}\left(y_{g}(k)-y(k)\right) .
$$

With the application of error function to derivate $W_{1}, W_{2}$, $W_{3}$, and $W_{4}$, we know the learning expression by using the gradient descent algorithm:

$$
\begin{gathered}
\Delta W_{j l}^{1}=\eta_{1} \sum_{i=1}^{m}\left(\delta_{i}^{0} W_{i j}^{3}\right) \frac{\partial X_{c 1 j}(k)}{\partial W_{j l}^{1}} \\
(j=1,2 \cdots n ; l=1,2 \cdots m), \\
\Delta W_{j q}^{2}=\eta_{2} \delta_{j}^{h} U_{q}(k-1), \quad(j=1,2 \cdots n ; q=1,2 \cdots r), \\
\Delta W_{i j}^{3}=\eta_{3} \delta_{i}^{0} Y_{h j}(k), \quad(j=1,2 \cdots m ; j=1,2 \cdots n), \\
\Delta W_{j l}^{4}=\eta_{4} \sum_{i=1}^{m}\left(\delta_{i}^{0} W_{i j}^{3}\right) \frac{\partial X_{c 2 j}(k)}{\partial W_{j l}^{4}} \\
(j=1,2 \cdots n ; l=1,2 \cdots m) .
\end{gathered}
$$

In the formulas, $\eta_{1}$ is learning step-size of $W^{1}, \eta_{2}$ of $W^{2}$, $\eta_{3}$ of $W^{3}$, and $\eta_{4}$ of $W^{4}$, and $\delta_{i}^{0}, \delta_{j}^{h}, \partial X_{c 2 j}(k) / \partial W_{j s}^{4}$, and $\partial X_{c 1 j}(k) / \partial W_{j l}^{1}$ are relevant functions. We can get the following expressions:

$$
\begin{gathered}
\delta_{i}^{0}=\left(Y_{g, i}(k)-Y_{i}(k) g_{i}^{\prime}(\cdot)\right), \\
\delta_{i}^{h}=\sum_{i=1}^{m}\left(\delta_{i}^{0} W_{i j}^{3}\right) f_{j}^{\prime}(\cdot), \\
\frac{\partial X_{c 1 j}(k)}{\partial W_{j l}^{1}}=f_{j}^{\prime}(\cdot) X_{c 1 j}(k-1)+\alpha \frac{\partial X_{c 1 j}(k-1)}{\partial W_{j l}^{1}}, \\
\frac{\partial X_{c 2 j}(k)}{\partial W_{j l}^{4}}=f_{j}^{\prime}(\cdot) X_{c 2 j}(k-1)+\alpha \frac{\partial X_{c 2 j}(k-1)}{\partial W_{j l}^{4}} .
\end{gathered}
$$

To avoid the problem of local optimal solution coming with local regression, comparison of predictive results is made between training samples and samples without training. If the predictive results are in the range of error threshold, they can be accepted. If not, they must be retrained until meeting the condition.

In the process of prediction, learning objects are defined as the nearest sample. In the set cycle, we update the network to realize the real-time control. The length of training samples is determined by control accuracy and predictive length in the system.

4.3. Control of Emulsion Pressure. Considering motion order of hydraulic support, combining feedback difference of pressure sensor, we get dynamic compensation of feeding pressure. The process is shown in Figure 3. 


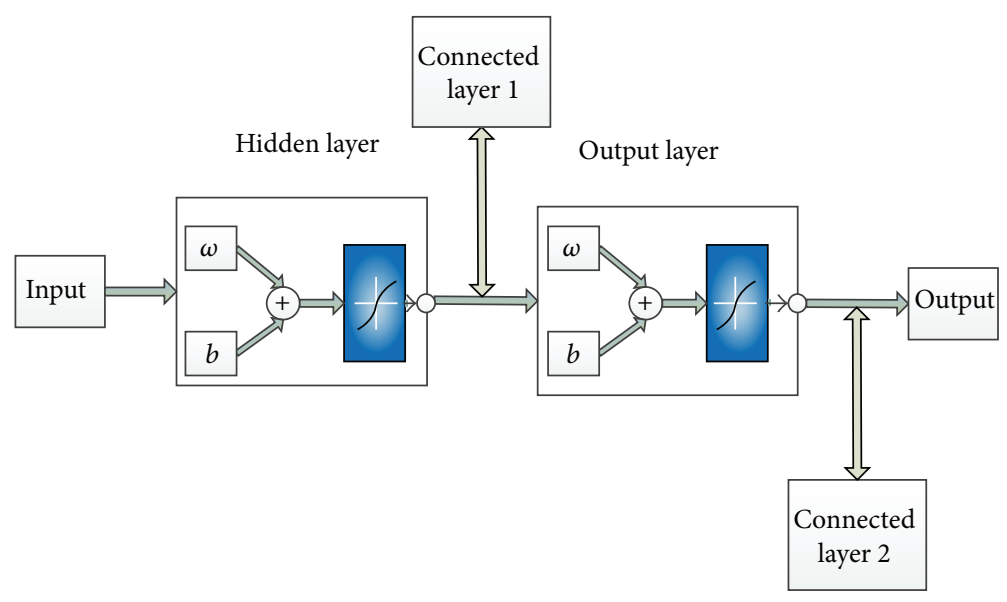

FIGURE 2: Principle of Elman neural network.

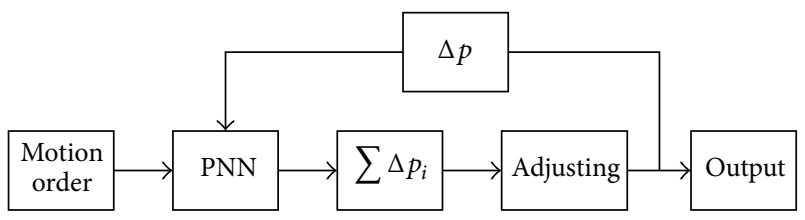

FIGURE 3: Dynamic compensation of feeding pressure.

In Figure 3, pressure sensor is located to detect oil pressure. And $\Delta p$ is difference value of detected value and expected output. In this paper, output pressure could be stabilized around $28 \mathrm{MPa}$ which is our controlling aim. $\sum p_{i}$ means sum influence value of hydraulic support motions in the next moment. And then we can adjust corresponding frequency to get the output.

According to regular hydraulic support motion, motion order is known, that is, retracting top coal-wall support, lowering the column, hauling support, raising the column, extending top coal-wall support, and then propelling and pushing.

4.4. The Flowchart of Proposed Approach. According to the above description about the approach based on Elman neural network, the proposed approach can be coded easily on the computer, and the flowchart can be summarized as shown in Figure 4.

\section{A Simulation Example and Industrial Experiment}

In this section, an engineering application of emulsion pump station pressure in a coal mine was put forward as a simulation example to verify the feasibility and effectiveness of proposed approach.

We chose six hydraulic supports as research objects. When hydraulic supports were running, the pressure of emulsion pump was detected. According to state coding of Table 1, we made statistics of motions and pressure. As the input layer, 5040 groups of data, gotten from the statistics, were shown in Table 2.
TABle 2: Part of input data.

\begin{tabular}{lccccccc}
\hline Coding & $\begin{array}{c}\mathrm{D} \text {-valve } \\
(\mathrm{MPa})\end{array}$ & $\Delta p_{1}$ & $\Delta p_{2}$ & $\Delta p_{3}$ & $\Delta p_{4}$ & $\Delta p_{5}$ & $\Delta p_{6}$ \\
\hline 000001 & +0.7 & +0.7 & 0 & 0 & 0 & 0 & 0 \\
000002 & +1.5 & 0 & +1.5 & 0 & 0 & 0 & 0 \\
000003 & +1.2 & 0 & 0 & +1.2 & 0 & 0 & 0 \\
000004 & -2 & 0 & 0 & 0 & -2 & 0 & 0 \\
000005 & +0.7 & 0 & 0 & 0 & 0 & +0.7 & 0 \\
000006 & -1 & 0 & 0 & 0 & 0 & 0 & -1 \\
000011 & +1.5 & +0.75 & 0 & 0 & 0 & 0 & 0 \\
000012 & +2 & +0.6 & +1.4 & 0 & 0 & 0 & 0 \\
000013 & +2 & +0.75 & 0 & +1.25 & 0 & 0 & 0 \\
$\vdots$ & $\vdots$ & $\vdots$ & $\vdots$ & & $\vdots$ & $\vdots$ & \\
555555 & +4.0 & 0 & 0 & 0 & 0 & +0.65 & 0 \\
666666 & -5.7 & 0 & 0 & 0 & 0 & 0 & -0.95 \\
\hline
\end{tabular}

About the coding, the first figure meant the state of the first hydraulic support. Likewise, the second figure meant the state of hydraulic support 2.

Input of the network was motion codes and their pressure differences, and output was every motion difference value in the condition. That is, we could get continuous updated motion difference valve. Through six hidden layer nodes, the network was well trained and the error was controlled in 5\%.

Emulsion pump pressure was influenced by both hydraulic support pressure and its motions. The proposed method was applied to emulsion pump control. The schematic diagram of control method was shown in Figure 5 and control results were shown in Figures 7 and 8.

And industrial experiment was described in detail as follows.

Step 1. Open emulsion pump station system and hydraulic system, and adjust emulsion pump station and hydraulic supports to condition of state. All of the system pipelines were normal, and no loss existed. 


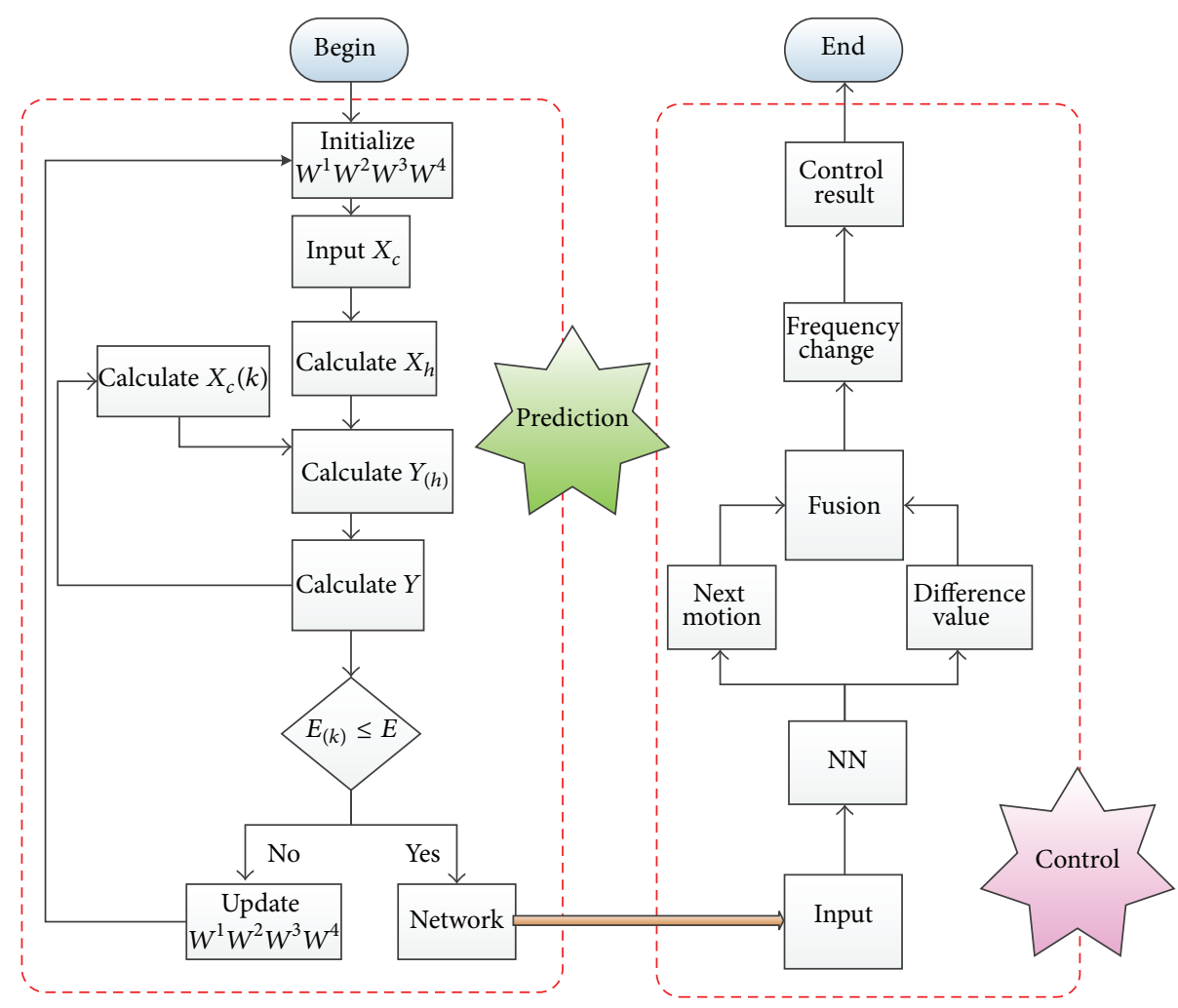

FIgURE 4: The flowchart of proposed approach.

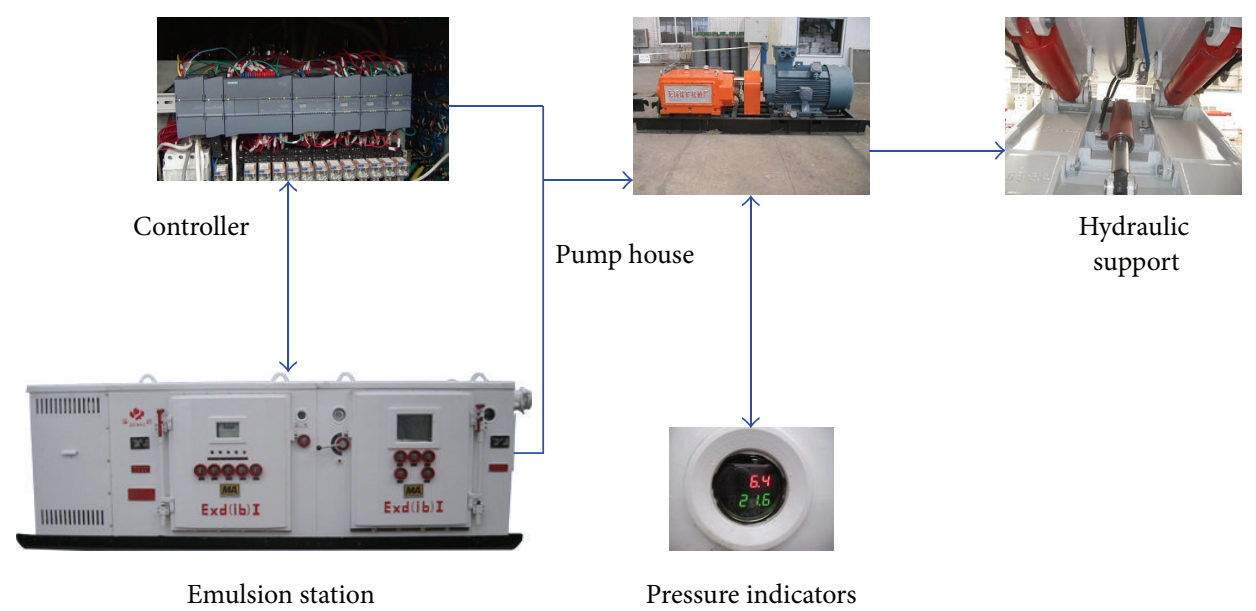

FIGURE 5: The schematic diagram of control method.

Step 2. Operations were done to the six hydraulic supports in five minutes, so actions were done in turns. The order of actions was as described in Table 1.

Step 3. After six actions, the six hydraulic supports would do the following motion combinations, which were $12,13,14,15$, $16,23,24,25,26,34,35,36,45,46,56,123,234,345,456$, and so on.

Step 4. Statistical analysis was done to experimental data in five minutes. Sampling period was 1 second.
In the controlling initial period, we got result in Figure 6. For a substantial time, the system became stable in Figure 7.

Analyzing control result, this controller could provide reasonable pressure to hydraulic motions. The proposed control method decreased pressure range which avoided frequent speed changing converter.

Figures 8 and 9 showed control results of PID and fuzzy PID controller [32]. Obviously, the proposed controller had better dynamic tracking property. Max fluctuation in the process of liquid turned smaller which reduced pipe wearing. 


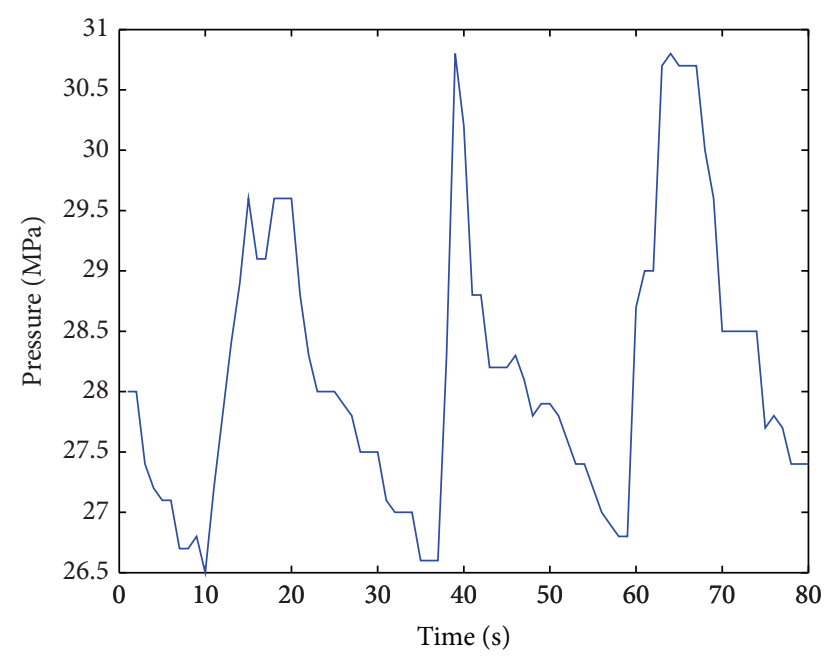

Figure 6: Control result based on hydraulic support motions.

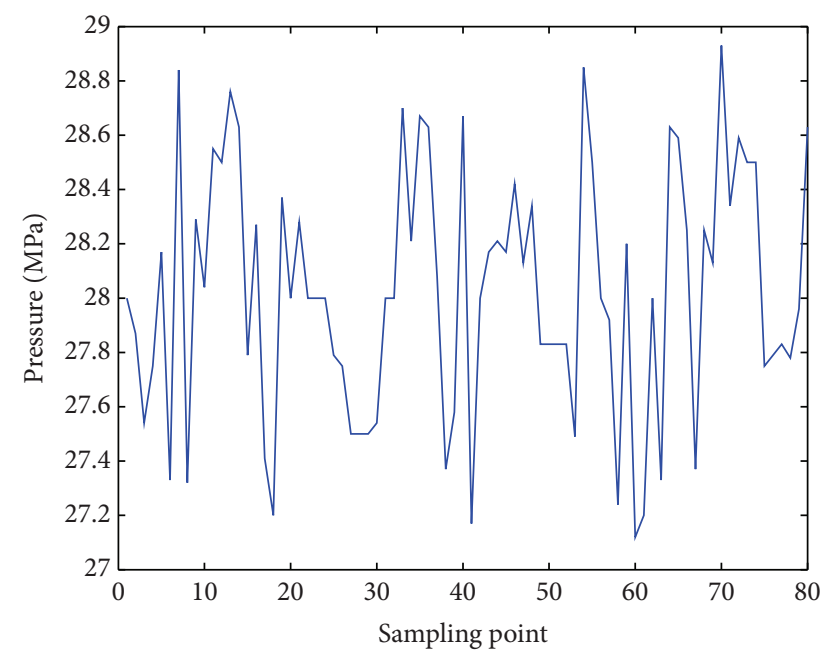

FIGURE 7: Stable result based on hydraulic support motions.

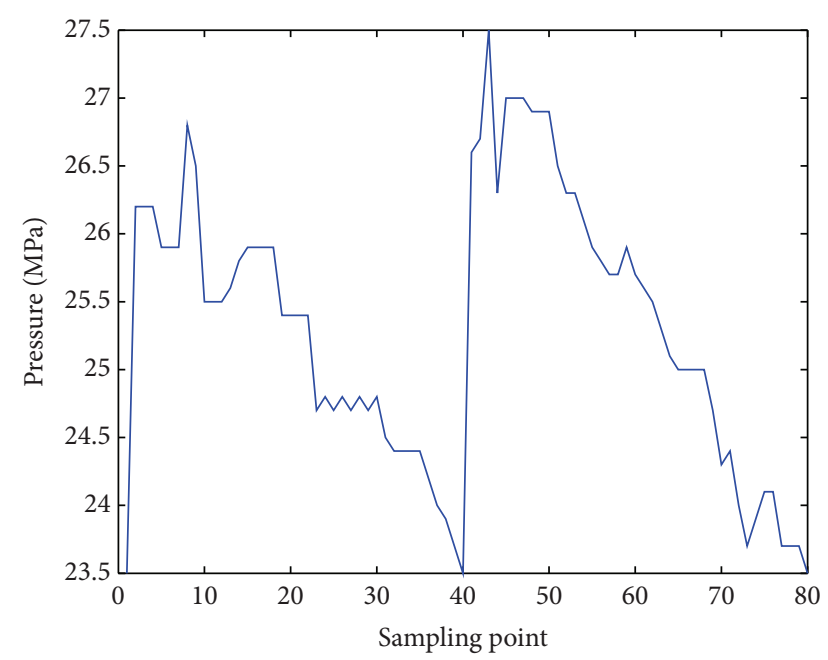

Figure 8: Control result of PID controller.

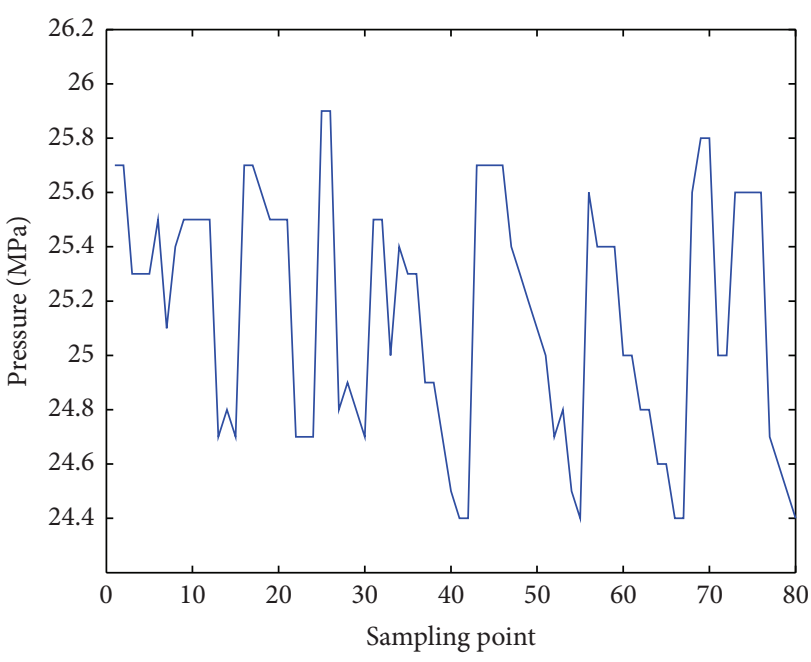

Figure 9: Control result of fuzzy PID controller.

TABLE 3: Control system specifications of three methods.

\begin{tabular}{lccc}
\hline Parameter & PID control & Fuzzy PID & Prediction control \\
\hline Range/MPa & $23.5 \sim 27.5$ & $24.4 \sim 25.9$ & $27.12 \sim 28.93$ \\
Mean value/MPa & 25.295 & 25.204 & 28.026 \\
Variance/MPa & 1.056 & 0.448 & 0.248 \\
Max error $/ \mathrm{MPa}$ & 4.5 & 3.6 & 0.93 \\
Relative coefficient & 0.02 & -0.07 & 0.63 \\
\hline
\end{tabular}

Smaller control error was found in prediction control method. In fact, as control methods based on PID ignored pressure loss in pipeline, control error would be larger along with longer pipeline. Control system specifications of three methods were shown in Table 3.

Direct measurement of response time was hard to do. Through calculating relative coefficient of experimental data and goal valve from the following equation, we got relative coefficients in Table 3 . In the equation, $r$ means relative coefficient. $x_{i}$ and $y_{i}$ mean $n$th of two sets of data:

$$
r=\frac{\sum_{i=1}^{n}\left(x_{i}-\bar{x}\right)\left(y_{i}-\bar{y}\right)}{\sqrt{\sum_{i=1}^{n}\left(x_{i}-\bar{x}\right)^{2} \cdot \sum_{i=1}^{n}\left(y_{i}-\bar{y}\right)^{2}}} .
$$

If goal valve of last second was shown in this second, we calculated that the relative coefficient was 0.23 . While relative coefficient of prediction control method was 0.63 , that was bigger than 0.23 . Obviously response time was less than 1 second.

\section{Conclusions and Future Work}

This paper proposed a control method for emulsion pressure based on Elman neural network considering hydraulic support motions. In order to verify the feasibility and superiority, the proposed approach was applied to an engineering problem of emulsion pressure control. The results of comparison simulations showed that the proposed approach could provide more reasonable pressure than existing PID controller. 
At the same time, the proposed method could help to save energy and make a contribution to protecting the environment. The control performance of proposed model demonstrated that this method could be extended to process other types of other pressure control.

In further studies, the authors plan to investigate control system considering more intelligent algorithms.

\section{Conflict of Interests}

The authors declare that there is no conflict of interests regarding the publication of this paper.

\section{Acknowledgments}

The support of The National Key Basic Research Program of China (973 Program): Key Fundamental Research on the Unmanned Mining Equipment in Deep Dangerous Coal Bed (2014CB046301), National High Technology Research and Development Program of China (no. 2013AA06A411), Xuyi Mine Equipment and Materials R\&D Center Innovation Fund Project (no. CXJJ201306), Jiangsu Province Ordinary University Graduate Student Scientific Research Innovation Project (KYLX_1373), and A Project Funded by the Priority Academic Program Development of Jiangsu Higher Education Institutions in carrying out this research is gratefully acknowledged.

\section{References}

[1] J. Su, Research on constant pressure control system in the emulsion pump station [Dissertation], Taiyuan University of Technology, 2012.

[2] I. S. Landet, A. Pavlov, and O. M. Aamo, "Modeling and control of heave-induced pressure fluctuations in managed pressure drilling," IEEE Transactions on Control Systems Technology, vol. 21, no. 4, pp. 1340-1351, 2013.

[3] R. Wang, "The apply of the adjustable modifying factor fuzzyPID Control in constant pressure water supply system," in Advances in Electronic Engineering, Communication and Management, pp. 25-28, Springer, Berlin, Germany, 2012.

[4] P. B. De Moura, J. Durães, and E. J. Solteiro Pires, "Mean arterial pressure PID control using a PSO-BOIDS algorithm," in International Joint Conference SOCO'13-CISIS'13-ICEUTE'13, Advances in Intelligent Systems and Computing, pp. 91-99, Springer, Berlin, Germany, 2014.

[5] T. K. Vrana and C. Hille, "A novel control method for dispersed converters providing dynamic frequency response," Electrical Engineering, vol. 93, no. 4, pp. 217-226, 2011.

[6] Y. Yuan, S. Tu, F. Zuo, and Q. Wu, "Support instability mechanism of fully mechanized top coal caving face with steep coal seams and its control," Journal of Mining \& Safety Engineering, no. 4, pp. 430-434, 2008.

[7] F. Meng, L. Xi, and G. Geng, "Research on pressure control method of airport pipeline fueling system based on selfadaptive fuzzy control," in Mechanical Engineering and Technology, T. Zhang, Ed., vol. 125 of Advances in Intelligent and Soft Computing, pp. 711-716, Springer, Berlin, Germany, 2012.
[8] T. Cai and H. Li, "Energy-saving emulsion pump automatic control system," in Proceedings of the 2nd International Conference on Intelligent Systems Design and Engineering Applications (ISDEA '12), pp. 829-831, IEEE, Hainan, China, January 2012.

[9] W.-Y. Li and J.-H. Wei, "Control strategy for multi-pump emulsion power station in coal mine face," Journal of Coal Science and Engineering, vol. 17, no. 4, pp. 443-446, 2011.

[10] K. Yang, L. Oh, and I. Lee, "Pressure control of hydraulic servo system using proportional control valve," KSME International Journal, vol. 13, no. 3, pp. 229-239, 1999.

[11] D. Zhang, C.-J. Zhang, Q. Wei, Y.-B. Tian, J.-B. Zhao, and X.-M. Li, "Modeling and control of piezo-stage using neural networks," Optics and Precision Engineering, vol. 20, no. 3, pp. 587-596, 2012.

[12] M. Harb, R. Abielmona, and E. Petriu, "Speed control of a mobile robot using neural networks and fuzzy logic," in Proceedings of the International Joint Conference on Neural Networks (IJCNN '09), pp. 1115-1121, Atlanta, Ga, USA, June 2009.

[13] Q. Zhu, S. Fei, T. Zhang, and T. Li, "Adaptive RBF neuralnetworks control for a class of time-delay nonlinear systems," Neurocomputing, vol. 71, no. 16-18, pp. 3617-3624, 2008.

[14] J. F. de Canete, J. Luque, J. Barbancho, and V. Muñoz, "Objectoriented modeling and simulation of the arterial pressure control system by using MODELICA," in Proceedings of the 13th Mediterranean Conference on Medical and Biological Engineering and Computing, pp. 923-926, Seville, Spain, September 2013.

[15] H. Yu and H. Li, "The energy saving research and design of automatic control of emulsion pump station," in Proceedings of the International Conference on Computational and Information Sciences (ICCIS '11), pp. 967-970, Chengdu, China, October 2011.

[16] T. Qiao and L. Yan, "Fuzzy-PID control and application in constant fluid feeding system," Coal Engineering, vol. 10, pp. 9597, 2011.

[17] B. Xu, H. Zhou, X. Shi, and J. Sun, "Design of frequencyconversion speed-regulation system of emulsion pump station," Industry and Mine Automation, no. 2, pp. 87-89, 2012.

[18] S. Yang, "Simulation of the unloading pressure and recovery pressure of internal unloading valve for emulsion pump station," Applied Mechanics and Materials, vol. 48-49, pp. 531-536, 2011.

[19] L. S. Mattos and D. G. Caldwell, "Capillary pressure control system for teleoperated and automatic biomanipulations," in $V$ Latin American Congress on Biomedical Engineering CLAIB 2011 May 16-21, 2011, Habana, Cuba, pp. 902-905, Springer, Berlin, Germany, 2013.

[20] R. Umrao and D. K. Chaturvedi, "A novel fuzzy control approach for load frequency control," in Recent Advancements in System Modelling Applications, pp. 239-247, 2013.

[21] B.-K. Yang, X.-Q. Bian, and W.-L. Guo, "Application of adaptive fuzzy control technology to pressure control of a pressurizer," Journal of Marine Science and Application, vol. 4, no. 1, pp. 3943, 2005.

[22] W. Tan, Y. Wang, S. Zhou, and Z. Liu, "Fuzzy neural network prediction of chaotic time series," Acta Physica Sinica, vol. 52, no. 4, pp. 795-801, 2003.

[23] L.-J. Yu and C.-C. Zhu, "Neural networks models on threshold electric field of field emission," Acta Physica Sinica, vol. 49, no. 1, pp. 170-172, 2000. 
[24] W. Junsong, W. Jiukun, Z. Maohua, and W. Junjie, "Prediction of internet traffic based on Elman neural network," in Proceedings of the Chinese Control and Decision Conference (CCDC '09), pp. 1248-1252, IEEE, Guilin, China, June 2009.

[25] E. Zhou, W. Yang, and J. Li, "Predictive control of hydraulic winch motion control," in Proceedings of the 2nd IEEE International Conference on Computer Science and Information Technology (ICCSIT '09), pp. 1-4, IEEE, Beijing, China, August 2009.

[26] L. Wang and X. Li, "Position sensorless control for PMLSM using Elman neural network," in Proceedings of the International Conference on Information Engineering and Computer Science (ICIECS '09), pp. 1-4, December 2009.

[27] J. L. Elman, "Finding structure in time," Cognitive Science, vol. 14, no. 2, pp. 179-211, 1990.

[28] X. Wang, C. Zhang, and S. Zhang, "Modified Elman neural network and its application to network traffic prediction," in Proceedings of the IEEE 2nd International Conference on Cloud Computing and Intelligent Systems (CCIS '12), pp. 629-633, 2012.

[29] S. Ding, Y. Zhang, J. Chen, and W. Jia, "Research on using genetic algorithms to optimize Elman neural networks," Neural Computing and Applications, vol. 23, no. 2, pp. 293-297, 2013.

[30] J.-T. Tang, Y. Cao, J.-Y. Xiao, and Q.-L. Guo, "Predication of plasma concentration of remifentanil based on Elman neural network," Journal of Central South University, vol. 20, no. 11, pp. 3187-3192, 2013.

[31] R. Güntürkün, "Determining the amount of anesthetic medicine to be applied by using elman's recurrent neural networks via resilient back propagation," Journal of Medical Systems, vol. 34, no. 4, pp. 493-497, 2010.

[32] W. Yao, G. Yu, and J. Song, "Pressure stabilization technology of high pressure and high flow emulsion pump station," Coal Science and Technology, vol. 40, no. 8, pp. 78-83, 2012. 

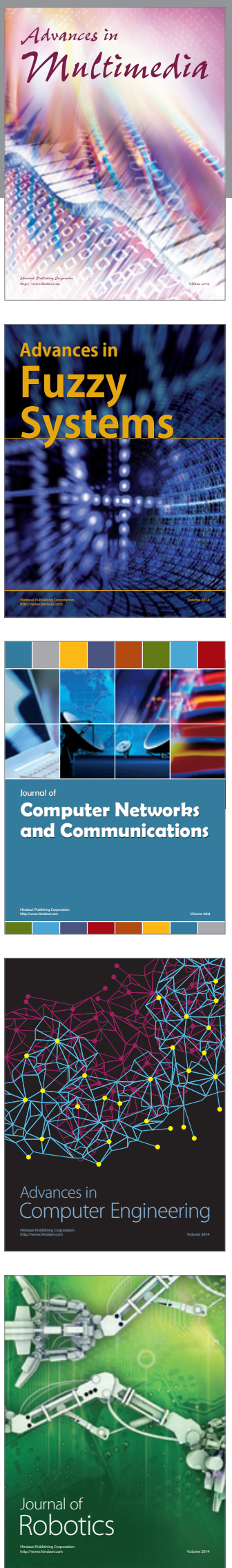

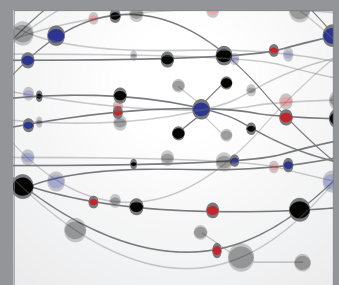

The Scientific World Journal
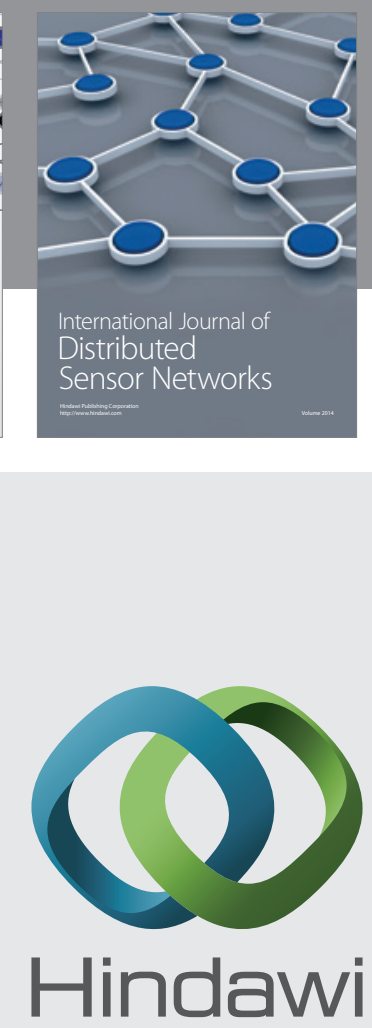

Submit your manuscripts at

http://www.hindawi.com
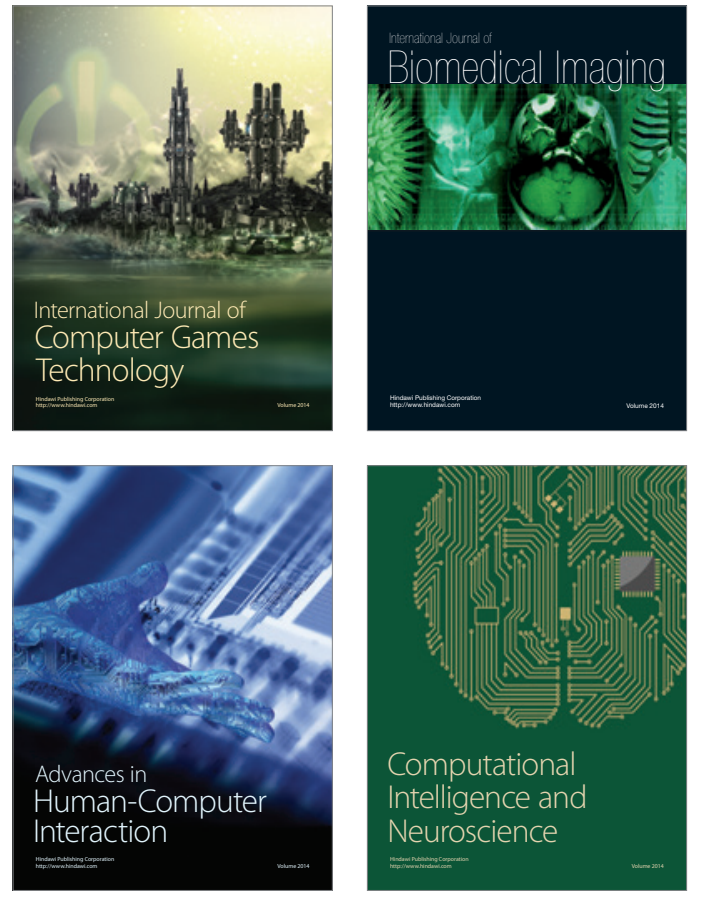
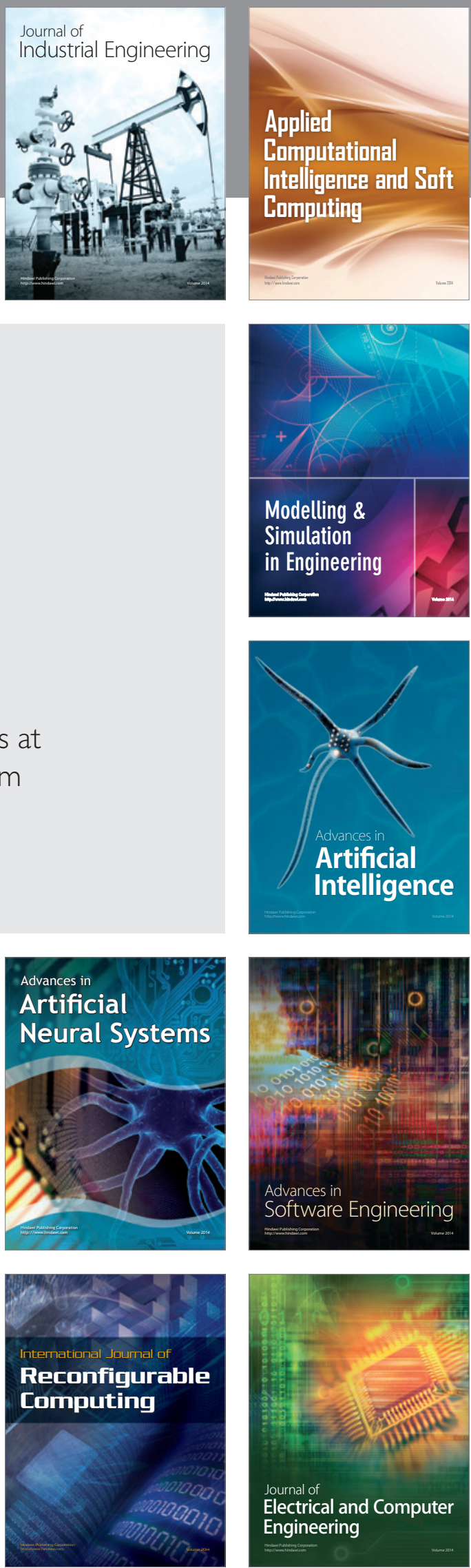